\title{
Communication Complexity of Distributed High Dimensional Correlation Testing
}

\author{
K. R. Sahasranand Himanshu Tyagi
}

\begin{abstract}
Two parties observe independent copies of a $d$-dimensional vector and a scalar. They seek to test if their data is correlated or not, namely they seek to test if the norm $\|\rho\|_{2}$ of the correlation vector $\rho$ between their observations exceeds $\tau$ or is it 0 . To that end, they communicate interactively and declare the output of the test. We show that roughly order $d / \tau^{2}$ bits of communication are sufficient and necessary for resolving the distributed correlation testing problem above. Furthermore, we establish a lower bound of roughly $d^{2} / \tau^{2}$ bits for communication needed for distributed correlation estimation, rendering the estimate-and-test approach suboptimal in communication required for distributed correlation testing. For the one-dimensional case with one-way communication, our bounds are tight even in the constant and provide a precise dependence of communication complexity on the probabilities of error of two types.
\end{abstract}

\section{INTRODUCTION}

Parties $\mathcal{P}_{1}$ and $\mathcal{P}_{2}$ observe jointly Gaussian random variables $X^{n}$ and $Y^{n}$, respectively, comprising independent and identically distributed (i.i.d.) samples $\left(X_{t}, Y_{t}\right), 1 \leq t \leq n$, with $X_{t} \in \mathbb{R}^{d}, Y_{t} \in \mathbb{R}$, and such that $\mathbb{E}\left[Y_{1} \mid X_{1}\right]=\rho^{T} X_{1}$. They communicate with each other to determine if their observations are correlated, i.e., to test if $\|\rho\|_{2} \geq \tau$ or $\|\rho\|_{2}=0$. For a given probability of error requirement and an arbitrary large $n$, what is the minimum communication needed between the parties?

Note that we have chosen the distribution to be Gaussian just for convenience. Since we allow the number of samples to be arbitrarily large, even when $X$ and $Y$ are not Gaussian, we can replace subset of samples with their sample means and use the central limit theorem (Berry-Esseen approximation) to do similar calculations as those presented in this paper. Indeed, all the results of this paper extend to the case when $X_{t}$ and $Y_{t}$ are distributed uniformly over $\{-1,1\}^{d}$ and $\{-1,1\}$, respectively, and

The authors are with the Department of Electrical Communication Engineering, Indian Institute of Science, Bangalore 560012, India. Email: \{sahasranand, htyagi\}@iisc.ac.in

A preliminary version of this paper [1] was presented at the IEEE International Symposium on Information Theory, Vail, USA, 2018. 
$\mathbb{E}\left[Y_{1} \mid X_{1}\right]=\rho^{T} X_{1}$. In another direction, it is seemingly restrictive to consider the conditional expectation to be a linear function of the observation vector $X_{1}$. However, as in nonparametric regression, we can express $E\left[Y_{1} \mid X_{1}=x_{1}\right]$ over an orthonormal basis for the $L_{2}$ space and replace the coordinates of $x_{1}$ with the evaluation of $f_{i}\left(x_{1}\right)$ for basis functions $f_{i}$. The dimension $d$ is chosen sufficiently large to capture most of the energy. In summary, our seemingly restrictive setup can be easily enhanced to the nonGaussian setting with nonlinear conditional expectation by using appropriate kernels (basis functions), as in nonparametric regression. In fact, this is the reason we call this problem correlation testing, instead of just independence testing.

This problem is an instance of a distributed hypothesis testing problem, which has been studied for several decades in the information theory literature starting with the seminal work [2] and closely followed by [3]. Most formulations in this literature focus on the tradeoff between the error exponent and communication rate per sample for simple binary hypothesis testing problems; see [4] for a survey. We remark that our setting differs from these classic settings since we consider a composite hypothesis testing problem. Furthermore, we do not focus on the error-exponent and allow arbitrarily large number of samples $n$. In particular, the error exponent can be shown to be 0 when we restrict to communication of rate $0(c f .[5])$, which is an allowed regime for us since we can take as many samples as we like to minimize the communication.

The problem of distributed independence testing with multiple rounds of interactive communication was studied in [6], [7]. Similar problems with more general hypotheses or more elaborate communication models have been considered in [8], [9], [10], [11]. Error exponent for the conditional independence testing problem is studied in [12], where both upper and lower bound for it are obtained. Recently, and subsequent to the publication of the initial version [1] of this paper, related problems were considered in various works. In [13], an improved upper bound on the Stein exponent for testing between two known positive Gaussian correlations is provided. The communication complexity of estimating one-dimensional Gaussian correlations was established in [14] and that of independence testing over discrete alphabet in the large sample regime was characterized in [15]. The tradeoff between communication complexity and sample complexity for detecting pairwise correlations is studied in [16]. A related line of recent work considers composite hypothesis testing under communication, privacy, and shared randomness constraints [17], [18], [19], [20]. However, the constraints are placed on each independent sample rather than on parties observing multiple correlated samples. In particular, none of the prior works consider our specific composite hypothesis testing problem.

The related problem of estimating the correlation vector for the same setting as ours was studied in [21]. It is plausible to use the distributed estimation scheme of [21] or similar estimation schemes to 
do testing, but we establish a lower bound to show that this approach will be suboptimal in communication requirement. Our main result is the characterization of the minimum communication needed for distributed correlation testing. Our proposed distributed test uses one-way communication and solves the $d$-dimensional problem by reducing it to the case $d=1$. This is done by multiplying the observation vectors $X_{t}$ s of $\mathcal{P}_{1}$ with a scaled random sign vector. Specifically, for $d=1$, our test entails the use of shared randomness to sample a vector that is close to $\mathcal{P}_{1}$ 's overall observation $\left(X_{1}, \ldots, X_{n}\right)$, sending the identity of this vector to $\mathcal{P}_{2}$, and then $\mathcal{P}_{2}$ checking if its observation vector $\left(Y_{1}, \ldots, Y_{n}\right)$ is close to this vector as well. We show that this test requires roughly $\max \left\{\left(1 / \tau^{2}\right) \log 1 / \varepsilon,\left(1 / \tau^{2}-1\right) \log 1 / \delta\right\}$ bits of communication to get probabilities of false alarm and missed detection to be less than $\delta$ and $\varepsilon$, respectively, when $n$ is sufficiently large. For a general $d$, noting that the multiplication with random sign will yield a one dimensional correlation testing problem with correlation roughly $\|\rho\|_{2} / \sqrt{d}$, we show that the $d$-dimensional problem can be resolved using roughly $\left(d / \tau^{2}\right) \max \{\log 1 / \varepsilon, \log 1 / \delta\}$ bits of communication.

Our proposed test is practical. In fact, we have simulated a version with slightly different parameters than those presented in our theoretical analysis below; the empirical performance is depicted in Figure 1. A phase transition in probability of error can be seen clearly when we communicate number of bits proportiona $\left.\right|^{1}$ to $d / \tau^{2}$.

Interestingly, we establish a lower bound that shows that the amount of one-way communication used by our protocol for $d=1$ is optimal among all one-way communication protocols. We show this bound by using the notions of hypercontractivity and reverse hypercontractivity ( $c f$. [22], [23], [24], [25], [26], [27]). Specifically, we note that the acceptance region corresponding to one-way communication corresponds to a union of disjoint rectangle sets. We use hypercontractivity to relate the measures of rectangle sets under the joint distribution corresponding to $|\rho|>\tau$ and the product distribution corresponding to $\rho=0$, which in turn leads to the required lower bound. In fact, by using the tensorization property we extend the bound to a general $d$ to obtain a lower bound on one-way communication of roughly $\left(d / \tau^{2}\right) \max \{\log 1 / \varepsilon, \log 1 / \delta\}$.

Recently, a strong data processing inequality for interactive correlation estimation was derived in [14]. We invoke this result to show that roughly $d / \tau^{2}$ bits of communication are needed even when interactive communication is allowed, rendering our proposed one-way communication protocol optimal among interactive protocols. We note that this bound is slightly weaker for one-way communication than the

\footnotetext{
${ }^{1}$ As will be seen below, our proposed test uses a "median trick" to convert the one-dimensional test to a $d$-dimensional test. In our simulation, even the probabilities of correctness for the one-dimensional test are boosted to the desired levels by repeating the tests and using a similar "median trick".
} 


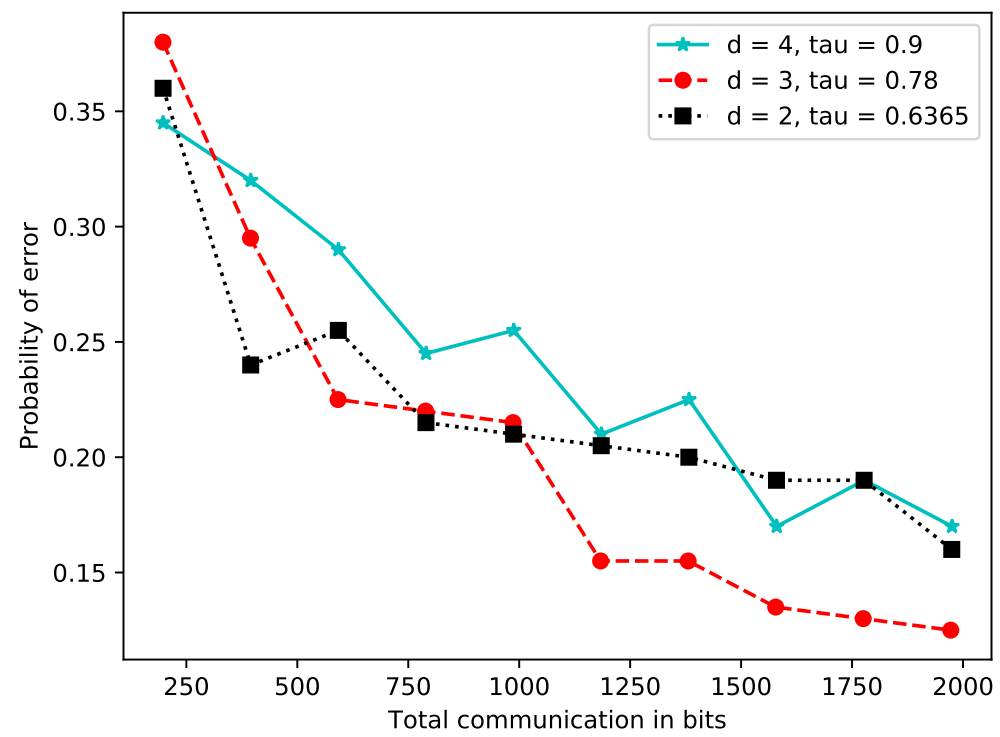

Fig. 1. Performance of the $d$-dimensional test for different values of $d$ and $\tau^{2}$ with $d / \tau^{2} \approx 4.93$. The probability of error (in $y$-axis) is the average of probability of false alarm and probability of missed detection, evaluated by averaging over 100 iterations.

one obtained using hypercontractivity.

As mentioned earlier, the related problem of correlation vector estimation was studied in [21]. In that work, an estimation protocol was given that uses roughly $d^{2} / \tau^{2}$ bits of communication to estimate $\rho$ within a mean squared error of $\tau$. Clearly, directly using this estimate to test will not be communication optimal. However, a natural question arises: can we find a better distributed estimation protocol that will remain communication-optimal even for testing? We show that, in fact, $d^{2} / \tau^{2}$ bits of communication are necessary for estimation, whereby estimate-and-test strategy is strictly suboptimal for testing.

We note that our proposed one-way communication scheme is related closely to the scheme in [28] where communication for common randomness generation ( $c f$. [29]) was considered. We draw on the heuristic connection between independence testing and common randomness generation highlighted in [30], [31] to adapt the scheme of [28] to devise a distributed correlation test.

We remark that a simple scheme is possible for $d=1$ that quantizes each value $X_{t}$ to its sign $\mathbb{1}\left\{X_{t} \geq 0\right\}$ and uses the known sample complexity results for independence testing for the collocated case ( $c f$. [32]). This, too, will result in a scheme that requires $O\left(1 / \tau^{2}\right)$ bits of communication. However, we noted in [1], where we study the communication complexity of one-dimensional independence testing, that our proposed scheme uses communication that is a constant factor lower that this baseline scheme. On the other hand, our scheme requires a much larger $n$ than this baseline scheme for $d=1$. 
The remainder of the paper is organized as follows. We present our problem formulation in the next section, followed by the main results in Section III. Our distributed correlation test as well as its analysis is presented in Section IV. The proof of our lower bounds for one-way communication is in Section $\mathrm{V}$ and for interactive communication is in Section VI, We conclude with a discussion and some extensions of our results in the final section.

Notation. Random variables are denoted by capital letters such as $X, Y$, etc.; their specific realizations by the corresponding small letters such as $x, y$, etc.; and their ranges by the corresponding calligraphic forms such as $\mathcal{X}, \mathcal{Y}$, etc.. $[N]$ denotes the set of integers $\{1,2, \ldots, N\}$. For a distribution $\mathbb{P}_{\rho}$ parametrized by $\rho$, we use $\mathbb{E}_{\rho}[X]$ to denote the expectation of the random variable $X$ with respect to $\mathbb{P}_{\rho} . \mathbb{P}_{\mathcal{H}_{i}}(A)$ denotes the probability of event $A$ under hypothesis $\mathcal{H}_{i}$. All the logarithms are to the base 2 ; when needed, we use $\ln a$ to denote the natural logarithm of $a$. For a vector $a, a(i)$ denotes the $i$-th coordinate, $a^{T}$ denotes the transpose, and $\|a\|_{p}:=\left(\sum_{i=1}^{d}|a(i)|^{p}\right)^{\frac{1}{p}}$ denotes the $\ell_{p}$-norm.

\section{PROBLEM SETUP}

We consider jointly Gaussian random variables $X \in \mathbb{R}^{d}$ and $Y \in \mathbb{R}$ with joint distribution as follows: for $\rho(i) \in[-1,1], 1 \leq i \leq d$, we assume that

$$
\begin{aligned}
& \mathbb{E}[X(i)]=0, \quad \mathbb{E}[X(i) X(j)]=\mathbb{1}\{i=j\}, 1 \leq i \leq j \leq d, \\
& \mathbb{E}[Y \mid X]=\sum_{i=1}^{d} \rho(i) X(i), \quad \mathbb{E}\left[Y^{2}\right]=1 .
\end{aligned}
$$

Note that the assumptions above imply $\mathbb{E}[Y]=0$. Since we assume $\mathbb{E}\left[Y^{2}\right]=1$, Jensen's inequality gives

$$
\|\rho\|_{2}^{2}=\mathbb{E}\left[\mathbb{E}[Y \mid X]^{2}\right] \leq \mathbb{E}\left[Y^{2}\right]=1
$$

Alternatively, we can describe the joint distribution of $X$ and $Y$ as follows:

$$
Y=\rho^{T} X+\sqrt{1-\|\rho\|_{2}^{2}} Z
$$

where $Z$ is a standard normal random variable, and $X$ and $Z$ are independent.

Let $\left(X_{t}, Y_{t}\right)_{t=1}^{n}$ denote $n$ independent copies of $(X, Y)$. We consider a distributed hypothesis testing problem where parties $\mathcal{P}_{1}$ and $\mathcal{P}_{2}$ observe $X^{n}=\left(X_{1}, \ldots, X_{n}\right)$ and $Y^{n}=\left(Y_{1}, \ldots, Y_{n}\right)$, respectively, and seek to resolve the following composite hypothesis testing problem:

$$
\begin{aligned}
& \mathcal{H}_{0}^{d}:\|\rho\|_{2} \geq \tau, \\
& \mathcal{H}_{1}^{d}: \rho=0,
\end{aligned}
$$


where $\tau$ takes values in $(0,1]$ and is known to both the parties.

To determine the true hypothesis, the parties communicate with each other interactively in multiple rounds. Specifically, the parties use an $r$-round interactive communication protocol $\pi$ that comprises mappings $f_{1}, \ldots, f_{r} ; \mathcal{P}_{1}$ and $\mathcal{P}_{2}$ use mappings $f_{i}, 1 \leq i \leq r$, to communicate in odd and even rounds $i$, respectively. Each mapping $f_{i}$ takes as input the local observation of the party, the previously seen communication, and a shared random variable $V$ available to both the parties and outputs a binary string. Formally, denoting by $C_{j}$ the random binary string sent in round $j$, we have

$$
\begin{gathered}
f_{i}:\left(X^{n}, C_{1}, \ldots, C_{i-1}, V\right) \mapsto C_{i} \in\{0,1\}^{\ell_{i}}, \quad 1 \leq i \leq r, i \text { odd } \\
f_{i}:\left(Y^{n}, C_{1}, \ldots, C_{i-1}, V\right) \mapsto C_{i} \in\{0,1\}^{\ell_{i}}, \quad 1 \leq i \leq r, i \text { even, }
\end{gathered}
$$

where $\ell_{i}, 1 \leq i \leq r$, denotes the length of communication in round $i$. The overall random communication $\left(C_{1}, \ldots, C_{r}\right)$ is called the transcript of the protocol and is denoted by $\Pi$. Furthermore, we denote by $|\pi|$ the length $\sum_{i=1}^{r} \ell_{i}$ of the transcript of the protocol. For simplicity, we describe our formulation below only for odd $r$; the case of even $r$ can be handled similarly.

For an odd $r$, an $r$-interactive distributed test $T=(\pi, g)$ consists of an $r$-round interactive communication protocol $\pi$ and a decision mapping $g:\left(Y^{n}, \Pi, V\right) \mapsto\{0,1\}$. A distributed test $T=(\pi, g)$ constitutes an $(\ell, \delta, \varepsilon, \tau)$-test with observation length $n$ if $|\pi|=\ell$ and

$$
\begin{aligned}
& \mathbb{P}_{\mathcal{H}_{0}^{d}}\left(g\left(Y^{n}, \Pi, V\right)=1\right) \leq \delta, \text { and } \\
& \mathbb{P}_{\mathcal{H}_{1}^{d}}\left(g\left(Y^{n}, \Pi, V\right)=0\right) \leq \varepsilon .
\end{aligned}
$$

Our goal is to design a distributed test that communicates as few bits as possible, while possessing the desired probabilities of error. Formally, we seek bounds for the minimum communication for $d$ dimensional correlation testing, defined next.

Definition 1. Given $\delta, \varepsilon \in[0,1]$ and $\tau \in(0,1]$, the minimum $r$-round communication for $d$-dimensional correlation testing $C_{d}^{r}(\delta, \varepsilon, \tau)$ is the least $\ell$ such that there exists an $(\ell, \delta, \varepsilon, \tau)$-test $T=(\pi, g)$ with an $r$-round interactive communication protocol $\pi$, for all observations of length $n$ sufficiently large.

The minimum communication for $d$-dimensional correlation testing $C_{d}(\delta, \varepsilon, \tau)$ is the infimum over $r \in \mathbb{N}$ of $C_{d}^{r}(\delta, \varepsilon, \tau)$.

While we have formulated the problem for general $r$, our main focus in this work is the minimum communication $C_{d}^{1}(\delta, \varepsilon, \tau)$ for one-way communication protocols. We characterize the dependence of $C_{d}^{1}(\delta, \varepsilon, \tau)$ on $\varepsilon$ (respectively $\delta$ ), up to absolute multiplicative constants and additive constants that may 
depend on $\delta$ (respectively $\varepsilon$ ). Furthermore, we show that the dependence on $\varepsilon$ is optimal up to constant factors, even when additional rounds of interaction are available. We summarize our results formally in the next section. But before that we formulate the related problem of correlation estimation.

We consider the problem of estimating $\rho$ for the joint distribution given in (1). The observation of the parties and the $r$-round interactive communication protocol is defined as before; as above, we define the problem only for odd $r$. An $r$-interactive distributed estimate is a pair $(\pi, \widehat{\rho})$ where $\pi$ is an $r$-round interactive communication protocol and $\widehat{\rho}:\left(Y^{n}, \Pi, V\right) \mapsto \widehat{\rho}\left(Y^{n}, \Pi, V\right) \in[-1,1]^{d}$.

An $r$-interactive distributed estimate $(\pi, \widehat{\rho})$ constitutes an $(\ell, \tau)$-estimate if $|\pi| \leq \ell$ and

$$
\mathbb{E}_{\rho}\left\{\left\|\widehat{\rho}\left(Y^{n}, \Pi, V\right)-\rho\right\|_{2}^{2}\right\} \leq \tau^{2}
$$

where $\mathbb{E}_{\rho}$ denotes the expectation with respect to the distribution in (1).

Definition 2. Given $\tau \in(0,1]$, the minimum $r$-round communication for $d$-dimensional correlation estimation $\widetilde{C}_{d}^{r}(\tau)$ is the least $\ell$ such that there exists an $(\ell, \tau)$-estimate $T=(\pi, \widehat{\rho})$ with an $r$-round interactive communication protocol $\pi$, for all observations of length $n$ sufficiently large.

The minimum communication for $d$-dimensional correlation estimation $\widetilde{C}_{d}(\tau)$ is the infimum over $r \in \mathbb{N}$ of $\widetilde{C}_{d}^{r}(\tau)$.

In the next section, we will provide a lower bound for $\widetilde{C}_{d}(\tau)$, which establishes roughly that correlation estimation requires much more communication than correlation testing.

\section{MAIN RESULTS}

We have divided our results into three parts: upper bounds for $C_{d}^{1}(\delta, \varepsilon, \tau)$ achieved by our proposed scheme, a lower bound for $C_{d}^{1}(\delta, \varepsilon, \tau)$, and a lower bound for $C_{d}(\delta, \varepsilon, \tau)$ with $r>1$. These parts are presented in separate sections below. The upshot of our results is that our protocol with $r=1$ uses almost minimum communication not only among one-way communication protocols, but also among interactive protocols. Furthermore, we establish a lower bound for the correlation estimation protocol which shows that it requires strictly more communication than correlation testing.

\section{A. Upper bounds for $C_{d}^{1}(\delta, \varepsilon, \tau)$}

Our goal in this work is to handle high dimensional correlation testing. Interestingly, we establish a reduction which relates the high dimensional case to $d=1$ case. To state our general result, first we state the result for $d=1$. 
Theorem 1. For every $\delta, \varepsilon \in(0,1)$,

$$
\begin{aligned}
C_{1}^{1}(\delta, \varepsilon, \tau) \leq \frac{1}{\tau^{2}} & \left(\sqrt{\log \frac{1}{\varepsilon}}+\sqrt{\left(1-\tau^{2}\right) \log \frac{1}{\delta}}\right)^{2} \\
& +\ln \left(\frac{2}{\tau^{2}}\left(\sqrt{\ln \frac{1}{\varepsilon}}+\sqrt{\left(1-\tau^{2}\right) \ln \frac{1}{\delta}}\right)^{2}+1\right)+O\left(\sqrt{\log \frac{1}{\delta} \log \frac{1}{\varepsilon}}\right) .
\end{aligned}
$$

To extend this result to the case of general $d$, we convert the $d$-dimensional problem to the onedimensional problem as follows: Party $\mathcal{P}_{1}$ applies a random rotation (using common randomness $V$ ) to the observed vector $X$ to obtain $\widetilde{X}$. We show that the first coordinate $\widetilde{X}(1)$ of the resulting vector $\widetilde{X}$ and $Y$ have correlation coefficient roughly $(\tau / \sqrt{d})$ under $\mathcal{H}_{0}^{1}$ (with high probability) and correlation coefficient 0 under $\mathcal{H}_{1}^{1}$. Using this fact (and a reduction result provided in the next section), we get the following upper bound for $C_{d}^{1}(\delta, \varepsilon, \tau)$.

Theorem 2. There exists a positive constant $c>0$ such that for every $\delta, \varepsilon \in(0,1)$ we have

$$
C_{d}^{1}(\delta, \varepsilon, \tau) \leq c \cdot \frac{d}{\tau^{2}} \cdot \max \left\{\log \frac{1}{\delta}, \log \frac{1}{\varepsilon}\right\}+O\left(\ln \frac{d}{\tau^{2}}\right)
$$

where the second term has no dependence on $\delta$ or $\varepsilon$.

B. Lower bounds for $C_{d}^{1}(\delta, \varepsilon, \tau)$

Our lower bound for the case $d=1$ matches the upper bound of Theorem 1 up to additive terms of lower order to yield the following characterization for $C_{1}^{1}(\delta, \varepsilon, \tau)$.

Theorem 3. For $\delta \leq 1 / 2$ and $\varepsilon$ such that $\delta+\varepsilon^{\frac{1-\tau}{1+\tau}} \leq 1$, we have $\mathrm{2}^{2}$

$$
C_{1}^{1}(\delta, \varepsilon, \tau)=\frac{1}{\tau^{2}} \log \frac{1}{\varepsilon}+O_{\delta}\left(\sqrt{\log \frac{1}{\varepsilon}}\right),
$$

and for $\delta, \varepsilon \in(0,1 / 2)$, we have

$$
C_{1}^{1}(\delta, \varepsilon, \tau)=\frac{1-\tau^{2}}{\tau^{2}} \log \frac{1}{\delta}+O_{\varepsilon}\left(\sqrt{\log \frac{1}{\delta}}\right),
$$

where the notation $O_{x}$ denotes that the constant implied by $O$ depends on $x$.

The proof of this result uses the notions of hypercontractivity and reverse hypercontractivity and is given in Section $\mathrm{V}$.

\footnotetext{
${ }^{2}$ With an abuse of notation, the $O(x)$ notation for the additive error denotes that the upper and lower bounds differ by at most an $O(x)$ term.
} 
In fact, we can relate the $d$-dimensional problem to the one-dimensional problem by revealing extra information to $\mathcal{P}_{2}$ to obtain a matching lower bound for Theorem 2 , from which the next result follows.

Theorem 4. For $0<\tau \leq 1, \delta \in(0,1 / 2)$, and $\varepsilon$ such that $\delta+\varepsilon^{\frac{1-\tau}{1+\tau}} \leq 1$, we have

$$
C_{d}^{1}(\delta, \varepsilon, \tau)=\Theta\left(\frac{d}{\tau^{2}} \cdot \max \left\{\log \frac{1}{\varepsilon}, \log \frac{1}{\delta}\right\}\right) .
$$

We remark that the reduction of the general $d \geq 1$ case to the one-dimensional case used in the proof of lower bound differs from the reduction in the upper bound; we provide the proof in Section $\mathrm{V}$. Nevertheless, it is interesting that we obtain tight results by relating the high dimensional setting to the one-dimensional setting.

\section{Lower bounds for $r \geq 1$}

Our final set of results provide lower bounds even for the interactive setting, establishing the optimality of our proposed distributed test even among interactive tests. To derive this lower bound, we use a data processing inequality from [33], which was used in a similar context in [14]. In fact, using this technique we can even derive a lower bound for the high dimensional correlation estimation problem, showing that this problem requires orderwise higher communication in comparison to correlation testing.

We begin with the result for the correlation testing problem. Note that we only prove optimality in the dependence on $\varepsilon$, and not on $\delta$.

Theorem 5. For $\delta, \varepsilon, \tau \in(0,1)$, we have

$$
C_{d}(\delta, \varepsilon, \tau) \geq \frac{d}{\tau^{2}}\left((1-\delta) \log \frac{1}{\varepsilon}-1\right) .
$$

The proof is provided in Section VI-A

We note that while the lower bound above extends the bounds from the previous section to the interactive setting, it does not yield optimal constants for $d=1$ and $r=1$ unlike Theorem 3. In fact, we believe that even the lower bound in Theorem 17 yields a tight constant; the slackness in characterization of $C_{d}^{1}(\delta, \varepsilon, \tau)$ arises from our upper bound. Thus, the lower bound in Theorem 5 is weaker than those given in the previous section for $r=1$.

Recall that the lower bounds of the previous section are derived using the concepts of hypercontractivity and reverse hypercontractivity (which appeared in the preliminary version of this paper [1]). As mentioned above, the lower bound in Theorem 5 uses a related but different idea of strong data processing inequalities. In particular, the following bound was derived in [14] using a strong data processing inequality; the statement follows by combining Theorems 7.1 and 7.2 in [14]. 
Lemma 6. (see [14]) For $\rho \in[-1,1]^{d}$ and any interactive communication protocol $\pi$ with inputs $X^{n}$ and $Y^{n}$ for parties $\mathcal{P}_{1}$ and $\mathcal{P}_{2}$, respectively, we have

$$
D\left(\mathbb{P}_{\rho} \| \mathbb{P}_{0}\right) \leq \rho_{\max }^{2}|\pi|,
$$

where $\rho_{\max }^{2}=\max _{i \in[d]} \rho(i)^{2}, \mathbb{P}_{\rho}$ denotes the distribution in (1) and $D(P \| Q)$ denotes the KullbackLeibler divergence between distributions $P$ and $Q$.

Using this bound and Fano's inequality, we derive the following lower bound for $\widetilde{C}_{d}(\tau)$.

Theorem 7. There exists a constant $c>0$, such that for every $\tau \in(0,1)$,

$$
\widetilde{C}_{d}(\tau) \geq \frac{c d^{2}}{\tau^{2}}
$$

The proof is provided in Section VI-B.

In fact, the lower bound above is tight too, and matches the upper bound attained by the distributed estimate proposed in [21]. The lower bound above establishes that a simple estimate-and-test approach using the estimate in [21] or other estimates will not be able to attain the optimal $O\left(d / \tau^{2}\right)$ communication needed for correlation testing.

\section{OUR SCHEME AND ITS ANALYSIS}

Our general scheme is obtained by first relating the $d$-dimensional correlation testing problem to the one-dimensional correlation testing problem, and then relating the one-dimensional problem to its onesided version. We develop a test for this one-sided problem first and then, in steps, convert it to a test for the $d$-dimensional problem in separate subsections below.

\section{A. One-sided correlation test}

Consider the following one-sided variant of the correlation testing problem with $d=1$ :

$$
\begin{aligned}
& \mathcal{H}_{0}^{+}: \rho \geq \tau, \\
& \mathcal{H}_{1}^{1}: \rho=0,
\end{aligned}
$$

where $\tau \in(0,1]$ is known to both parties. We present a 1-interactive distributed test for this problem; namely, we present a test using one-way communication from $\mathcal{P}_{1}$ to $\mathcal{P}_{2}$.

Specifically, fix parameters $r>0, \theta \leq \tau$, and $k \in \mathbb{N}$. Throughout this section, for brevity, with a slight abuse of notation we denote by $X=\left(X_{1}, \ldots, X_{n}\right) \in \mathbb{R}^{n}$ and $Y=\left(Y_{1}, \ldots, Y_{n}\right) \in \mathbb{R}^{n}$, respectively, the 
observation of $\mathcal{P}_{1}$ and $\mathcal{P}_{2}$, where $\left(X_{t}, Y_{t}\right)_{t=1}^{n}$ are generated i.i.d. from the distribution in (1). Furthermore, for two vectors $u$ and $v$ in $\mathbb{R}^{n}$, we denote $u \cdot v:=u^{T} v$.

1) Using the shared randomness, parties generate an $n \times 2^{k}$ matrix $U$ consisting of i.i.d. uniform $\{-1,+1\}$-valued entries $U_{i j}, 1 \leq i \leq n, 1 \leq j \leq 2^{k}$.

2) Let $U_{j}$ denote the $j$-th column of $U$. $\mathcal{P}_{1}$ finds the least index $j \in\left[2^{k}\right]$ such that

$$
U_{j} \cdot X \geq r \sqrt{n}
$$

and sends the $k$-bit representation of $j$ to $\mathcal{P}_{2}$. If no such $j$ is found, declare $\mathcal{H}_{1}^{1}$.

3) $\mathcal{P}_{2}$, upon receiving $j$, declares $\mathcal{H}_{0}^{+}$if

$$
U_{j} \cdot Y \geq \theta \cdot r \sqrt{n}
$$

The next result captures the performance of our proposed distributed test.

Theorem 8. For $\delta, \varepsilon \in(0,1), \tau \in(0,1)$, an appropriate choice of $\theta \leq \tau$, and for all $n$ sufficiently large, the 1-interactive test proposed above satisfies

$$
\mathbb{P}_{\mathcal{H}_{0}^{+}}\left[\text {Declare } \mathcal{H}_{1}^{1}\right] \leq \delta \text { and } \mathbb{P}_{\mathcal{H}_{1}^{1}}\left[\text { Declare } \mathcal{H}_{0}^{+}\right] \leq \varepsilon
$$

when $r$ is set as follows:

$$
r^{2}=\frac{2 \ln 2}{\tau^{2}}\left(\sqrt{\log \frac{1}{\varepsilon}+\log \ln \frac{3}{\delta}+1}+\sqrt{\left(1-\tau^{2}\right) \log \frac{3}{\delta}}\right)^{2}
$$

and the communication length $k$ satisfies

$$
k=\left\lceil\log \frac{1}{Q(r)}+\log \ln \frac{3}{\delta}\right\rceil,
$$

where $Q(\cdot)$ denotes the complementary cumulative distribution function of a standard Gaussian random variable.

Proof. We begin by deriving a lower bound for the probability of correctly declaring $\mathcal{H}_{0}^{+}$. We have

$$
\mathbb{P}_{\mathcal{H}_{0}^{+}}\left[\text {Declare } \mathcal{H}_{0}^{+}\right]=\sum_{j=1}^{2^{k}} \mathbb{P}_{\mathcal{H}_{0}^{+}}\left(U_{l} \cdot X<r \sqrt{n} \text { for all } l \leq j-1, U_{j} \cdot X \geq r \sqrt{n}, U_{j} \cdot Y \geq \theta \cdot r \sqrt{n}\right),
$$

where $U_{0}$ is set to be 0 . We approximate the right-side using the Berry-Esseen theorem (cf. [34]) for a fixed realization $X=x$. Specifically, noting that $U_{j} \cdot x=\sum_{i=1}^{n} U_{i j} x_{i}$ is a sum of independent random 
variables, the Berry-Esseen theorem yields

$$
\begin{aligned}
& \mathbb{P}_{\mathcal{H}_{0}^{+}}\left(U_{l} \cdot X<r \sqrt{n} \text { for all } l \leq j-1, U_{j} \cdot X \geq r \sqrt{n} \mid X=x\right) \\
& =\left[\mathbb{P}_{\mathcal{H}_{0}^{+}}\left(\sum_{i=1}^{n} U_{i 1} x_{i}<r \sqrt{n} \mid X=x\right)\right]^{j-1} \mathbb{P}_{\mathcal{H}_{0}^{+}}\left(\sum_{i=1}^{n} U_{i j} x_{i} \geq r \sqrt{n} \mid X=x\right) \\
& \geq\left(1-Q\left(\frac{r \sqrt{n}}{\sqrt{\sum_{i=1}^{n} x_{i}^{2}}}\right)-c_{0} \frac{\sum_{i=1}^{n}\left|x_{i}\right|^{3}}{\left(\sum_{i=1}^{n} x_{i}^{2}\right)^{\frac{3}{2}}}\right)^{j-1}\left(Q\left(\frac{r \sqrt{n}}{\sqrt{\sum_{i=1}^{n} x_{i}^{2}}}\right)-c_{0} \frac{\sum_{i=1}^{n}\left|x_{i}\right|^{3}}{\left(\sum_{i=1}^{n} x_{i}^{2}\right)^{\frac{3}{2}}}\right),
\end{aligned}
$$

where $c_{0}$ is a constant. Next, note that under $\mathcal{H}_{0}^{+}$, for each $i \in[n]$ we have $\mathbb{E}\left[Y_{i} \mid X_{i}\right]=\rho X_{i}$ with $\rho \geq \tau$. It follows that for a fixed realization $X=x$ and $U=u$, the random variables $U_{i j} Y_{i}, 1 \leq i \leq n$, are independent with distribution $\mathcal{N}\left(\rho x_{i}, 1-\rho^{2}\right)$ for every $j \in\left[2^{k}\right]$. Note that for $u_{j} \cdot x \geq r \sqrt{n}$, we have

$$
\mathbb{E}\left[U_{j} \cdot Y \mid U=u, X=x\right]=\rho\left(u_{j} \cdot x\right) \geq \rho r \sqrt{n} .
$$

Therefore, for every $u$ and $x$ such that $u_{j} \cdot x \geq r \sqrt{n}$ and $u_{l} \cdot x<r \sqrt{n}$ for all $l \leq j-1$, we have

$$
\mathbb{P}_{\mathcal{H}_{0}^{+}}\left(U_{j} \cdot Y \geq \theta r \sqrt{n} \mid U=u, X=x\right) \geq Q\left(\frac{r(\theta-\rho)}{\sqrt{1-\rho^{2}}}\right) \geq Q\left(\frac{r(\theta-\tau)}{\sqrt{1-\tau^{2}}}\right),
$$

where the final bound holds since $Q(a)$ is decreasing in $a$ and the function $f(a)=(\theta-a) / \sqrt{1-a^{2}}$ is non increasing in $a$ for $a \geq \theta$; specifically, this bound uses our assumption that $\theta \leq \tau$.

Upon combining the bounds above, denoting $\sigma_{n}(X)=\sqrt{\sum_{i=1}^{n} X_{i}^{2}}$ and $\beta_{n}(X)=c_{0} \sum_{i=1}^{n}\left|X_{i}\right|^{3} / \sigma_{n}^{3}(X)$, we obtain

$$
\begin{aligned}
& \mathbb{P}_{\mathcal{H}_{0}^{+}}\left[\text {Declare } \mathcal{H}_{0}^{+}\right] \\
& \geq \mathbb{E}\left[\frac{Q\left(\frac{r \sqrt{n}}{\sigma_{n}(X)}\right)-\beta_{n}(X)}{Q\left(\frac{r \sqrt{n}}{\sigma_{n}(X)}\right)+\beta_{n}(X)} \times\left(1-\left(1-Q\left(\frac{r \sqrt{n}}{\sigma_{n}(X)}\right)-\beta_{n}(X)\right)^{2^{k}}\right)\right] Q\left(\frac{r(\theta-\tau)}{\sqrt{1-\tau^{2}}}\right) .
\end{aligned}
$$

Using the law of large numbers and the inequality $1-a \leq e^{-a}$, for every $\eta>0$ and all $n$ sufficiently large, we get

$$
\begin{aligned}
\mathbb{P}_{\mathcal{H}_{0}^{+}}\left[\text {Declare } \mathcal{H}_{0}^{+}\right] & \geq(1-\eta)\left(1-e^{-2^{k} Q(r)}\right) Q\left(\frac{r(\theta-\tau)}{\sqrt{1-\tau^{2}}}\right) \\
& \geq 1-e^{-2^{k} Q(r)}-Q\left(\frac{r(\tau-\theta)}{\sqrt{1-\tau^{2}}}\right)-\eta
\end{aligned}
$$

where we used the bound $(1-x)(1-y)(1-z) \geq 1-(x+y+z)$ for $x, y, z \in(0,1)$.

Next, we derive an upper bound for the probability of declaring $\mathcal{H}_{0}^{+}$when $\mathcal{H}_{1}^{1}$ is true; we derive a bound for this probability which holds for every fixed realization $u$ of the random codebook $U$. Since 
$\sum_{i=1}^{n} u_{i j} X_{i}$ is a sum of $n$ independent standard Gaussian random variables, we have

$$
\mathbb{P}_{\mathcal{H}_{1}^{1}}\left(\sum_{i=1}^{n} u_{i j} X_{i} \geq r \sqrt{n} \mid U=u\right) \leq Q(r),
$$

and similarly,

$$
\mathbb{P}_{\mathcal{H}_{1}^{1}}\left(\sum_{i=1}^{n} u_{i j} Y_{i} \geq \theta r \sqrt{n} \mid U=u\right) \leq Q(\theta r) .
$$

Therefore,

$$
\begin{aligned}
\mathbb{P}_{\mathcal{H}_{1}^{1}}\left(\text { Declare } \mathcal{H}_{0}^{+}\right) & \leq \mathbb{E}_{U}\left[\sum_{j=1}^{2^{k}} \mathbb{P}_{\mathcal{H}_{1}^{1}}\left(\sum_{i=1}^{n} u_{i j} X_{i} \geq r \sqrt{n}\right) \cdot \mathbb{P}_{\mathcal{H}_{1}^{1}}\left(\sum_{i=1}^{n} u_{i j} Y_{i} \geq \theta r \sqrt{n}\right)\right] \\
& \leq 2^{k} Q(r) Q(\theta r) .
\end{aligned}
$$

To satisfy the error condition (3), by (4) and (5) it suffices to set $\eta=\delta / 3$ and choose $r, \theta$, and $k$ to satisfy the following:

$$
\begin{aligned}
\ln \frac{3}{\delta} & \leq 2^{k} Q(r) \leq 2 \ln \frac{3}{\delta}, \\
Q\left(\frac{r(\tau-\theta)}{\sqrt{1-\tau^{2}}}\right) & \leq \frac{\delta}{3}, \\
Q(\theta r) & \leq \frac{\varepsilon}{2 \ln \frac{3}{\delta}} .
\end{aligned}
$$

Using Chernoff bound $Q(x) \leq e^{-x^{2} / 2}$, for conditions (7) and (8) it suffices to have

$$
\begin{array}{r}
\frac{1-\tau^{2}}{(\tau-\theta)^{2}} \cdot \log \frac{3}{\delta} \leq \frac{r^{2}}{2 \ln 2}, \\
\frac{1}{\theta^{2}}\left(\log \frac{1}{\varepsilon}+\log \ln \frac{3}{\delta}+1\right) \leq \frac{r^{2}}{2 \ln 2} .
\end{array}
$$

Therefore, the least value of $k$ is given by an $r$ that satisfies

$$
\frac{r^{2}}{2 \ln 2}=\min _{\theta \leq \tau} \max \left\{\frac{a}{\theta^{2}}, \frac{b}{(\tau-\theta)^{2}}\right\}
$$

where $a=\left(\log \frac{1}{\varepsilon}+\log \ln \frac{3}{\delta}+1\right)$ and $b=\left(1-\tau^{2}\right) \log \frac{3}{\delta}$. The optimal $\theta^{*}$ for the problem on the right-side is given by

$$
\theta^{*}=\frac{\tau \sqrt{a}}{\sqrt{b}+\sqrt{a}}
$$

whereby our optimal choice of $r^{2}$ is

$$
\frac{r^{2}}{2 \ln 2}=\frac{1}{\tau^{2}}(\sqrt{a}+\sqrt{b})^{2}
$$




$$
=\frac{1}{\tau^{2}}\left(\sqrt{\log \frac{1}{\varepsilon}+\log \ln \frac{3}{\delta}+1}+\sqrt{\left(1-\tau^{2}\right) \log \frac{3}{\delta}}\right)^{2} .
$$

Thus, by (6), we can satisfy (3) if we $\left.\operatorname{set}^{2}\right\rceil k=\left\lceil\log \frac{1}{Q(r)}+\log \ln \frac{3}{\delta}\right\rceil$ for $r$ given above.

\section{B. Distributed correlation test for $d=1$}

We now extend the one-sided test above to a test for $d=1$. We present a general reduction which will allow us to use any 1-interactive distributed test for the one-sided problem (not just the one above) for the (two-sided) correlation testing problem with $d=1$.

Lemma 9 (Two-sided to one-sided). For $\delta \in(0,1), \varepsilon \in(0,1 / 2), \tau \in(0,1)$, and $\ell \in \mathbb{N}$, suppose that $T^{+}=T^{+}\left(X^{n}, Y^{n}\right)$ is an 1-interactive $(\ell, \delta, \varepsilon, \tau)$-test for the one-sided correlation testing problem. Then, we can find a 1-interactive $(\ell, \delta, 2 \varepsilon, \tau)$-test for the correlation testing problem with $d=1$.

Proof. We begin by noting that $T^{-}\left(X^{n}, Y^{n}\right)=T^{+}\left(X^{n},-Y^{n}\right)$ is an $(\ell, \delta, \varepsilon, \tau)$-test for the following alternative one-sided problem:

$$
\begin{aligned}
& \mathcal{H}_{0}^{-}: \rho \leq-\tau, \\
& \mathcal{H}_{1}^{1}: \rho=0 .
\end{aligned}
$$

Note that the communication protocol for $T^{+}$and $T^{-}$is the same; the corresponding decision mappings $g^{+}$and $g^{-}$differ. In particular, $g^{-}\left(Y^{n}, \Pi, V\right)=g^{+}\left(-Y^{n}, \Pi, V\right)$, and let $\pi$ be the common communication protocol for $T^{+}$and $T^{-}$. Consider the following 1-interactive distributed test $T=(\pi, g)$ for the correlation testing problem.

1) Parties execute the communication protocol $\pi$.

2) Use decision mapping $g\left(Y^{n}, \Pi, V\right)=\min \left\{g^{+}\left(Y^{n}, \Pi, V\right), g^{-}\left(Y^{n}, \Pi, V\right)\right\}$.

For this test, we can verify that

$$
\begin{aligned}
\mathbb{P}_{\mathcal{H}_{1}^{1}}\left[g\left(Y^{n}, \Pi, V\right)=0\right] & =\mathbb{P}_{\mathcal{H}_{1}^{1}}\left[g^{+}\left(Y^{n}, \Pi, V\right)=0 \text { or } g^{-}\left(Y^{n}, \Pi, V\right)=0\right] \\
& \leq \mathbb{P}_{\mathcal{H}_{1}^{1}}\left[g^{+}\left(Y^{n}, \Pi, V\right)=0\right]+\mathbb{P}_{\mathcal{H}_{1}^{1}}\left[g^{-}\left(Y^{n}, \Pi, V\right)=0\right] \\
& \leq 2 \varepsilon .
\end{aligned}
$$

Furthermore, under $\mathcal{H}_{0}^{1}$,

$$
\mathbb{P}_{\mathcal{H}_{0}^{1}}\left[g\left(Y^{n}, \Pi, V\right)=1\right]=\mathbb{P}_{\mathcal{H}_{0}^{1}}\left[g^{+}\left(Y^{n}, \Pi, V\right)=g^{-}\left(Y^{n}, \Pi, V\right)=1\right]
$$

\footnotetext{
${ }^{2}$ In our analysis, we cannot set $k$ higher than this either.
} 


$$
\begin{aligned}
& \leq \max \left\{\mathbb{P}_{\mathcal{H}_{0}^{+}}\left[g^{+}\left(Y^{n}, \Pi, V\right)=g^{-}\left(Y^{n}, \Pi, V\right)=1\right],\right. \\
& \left.\quad \mathbb{P}_{\mathcal{H}_{0}^{-}}\left[g^{+}\left(Y^{n}, \Pi, V\right)=g^{-}\left(Y^{n}, \Pi, V\right)=1\right]\right\} \\
& \leq \delta
\end{aligned}
$$

which shows that $T$ constitutes an $(\ell, \delta, 2 \varepsilon, \tau)$-test.

Lemma 9. Theorem 8, and the well-known bound $Q(x) \geq \frac{x}{\sqrt{2 \pi}\left(x^{2}+1\right)} e^{-x^{2} / 2}$ yield Theorem 1 .

\section{Proof of Theorem 2}

Finally, now that we have a correlation test for $d=1$, we complete the proof of Theorem 2 to obtain a test for general $d$. We begin by making a simple observation akin to the "median trick" in randomized algorithms.

Lemma 10. For $\alpha, \beta, \tau \in(0,1)$ with $\alpha+\beta<1$, suppose that we have an $r$-interactive $(\ell, \alpha, \beta, \tau)$ test for the $d$-dimensional correlation testing problem. Then, for every $\delta, \varepsilon \in(0,1)$, we can obtain an $r$-interactive $(m \ell, \delta, \varepsilon, \tau)$-test for the d-dimensional correlation testing problem whenever

$$
m \geq \frac{2}{(1-\beta+\alpha)^{2}} \max \left\{\ln \frac{1}{\delta}, \ln \frac{1}{\varepsilon}\right\} .
$$

Proof. We provide proof only for odd $r$; even $r$ can be handled similarly. Consider an $r$-interactive distributed test $T=(\pi, g)$ that satisfies

$$
\begin{aligned}
& \mathbb{P}_{\mathcal{H}_{0}^{d}}\left(g\left(Y^{n}, \Pi, V\right)=1\right) \leq \alpha, \\
& \mathbb{P}_{\mathcal{H}_{1}^{d}}\left(g\left(Y^{n}, \Pi, V\right)=0\right) \leq \beta,
\end{aligned}
$$

where $\pi$ is a communication protocol of length $\ell$. To construct the desired test, we repeat the test above $m$ times independently. Specifically, we first apply the test above to $m$ independent copies of $\left(X^{n}, Y^{n}, V\right)$ to obtain transcripts $\Pi_{1}, \ldots, \Pi_{m}$. Note that the resulting communication protocol is still an $r$-round protocol, with length $m \ell$. Denote by $V_{1}, \ldots, V_{m}$ the independent copies of the shared randomness used for the protocol. Further, denote by $D_{i}$ the output $g\left(Y_{n(i-1)+1}^{n i}, \Pi_{i}, V_{i}\right), 1 \leq i \leq m$, for the $i$-th copy of the test. Consider the new decision mapping $g^{m}$ given by

$$
g^{m}\left(Y^{n m}, \Pi^{m}, V^{m}\right)=\mathbb{1}\left\{\sum_{i=1}^{m} D_{i}>m t\right\},
$$


for a fixed $\alpha<t<1-\beta$. Note that $D_{1}, \ldots, D_{m}$ are independent bits and by our assumption about $T$, satisfy

$$
\begin{aligned}
& \mathbb{P}_{\mathcal{H}_{0}^{d}}\left(D_{i}=1\right) \leq \alpha, \\
& \mathbb{P}_{\mathcal{H}_{1}^{d}}\left(D_{i}=1\right) \geq 1-\beta,
\end{aligned}
$$

for every $1 \leq i \leq m$. Therefore, by Hoeffding's inequality,

$$
\mathbb{P}_{\mathcal{H}_{0}^{d}}\left(g^{m}\left(Y^{n m}, \Pi^{m}, V^{m}\right)=1\right)=\mathbb{P}_{\mathcal{H}_{0}^{d}}\left(\sum_{i=1}^{m} D_{i}>m t\right) \leq e^{-2 m(t-\alpha)^{2}},
$$

and similarly,

$$
\mathbb{P}_{\mathcal{H}_{1}^{d}}\left(g^{m}\left(Y^{n m}, \Pi^{m}, V^{m}\right)=0\right)=\mathbb{P}_{\mathcal{H}_{1}^{d}}\left(\sum_{i=1}^{m} D_{i} \leq m t\right) \leq e^{-2 m(1-\beta-t)^{2}} .
$$

In particular, by setting $m \geq \frac{2}{(1-\beta+\alpha)^{2}} \max \left\{\ln \frac{1}{\delta}, \ln \frac{1}{\varepsilon}\right\}$ and $t=(1-\beta+\alpha) / 2$, we obtain the desired test.

Thus, it suffices to construct a distributed test with constant probability of error. We do that in the result below by using a 1-interactive distributed test for $d=1$. Our test uses a randomized construction; to facilitate its analysis, we note the following fact.

Lemma 11. For $R=\frac{1}{\sqrt{d}} W$ with $W$ a random vector consisting of i.i.d. Rademacher entries, for every vector $x \in \mathbb{R}^{d}$ we have,

$$
\mathbb{P}\left(\left(R^{T} x\right)^{2} \geq \frac{\|x\|_{2}^{2}}{2 d}\right) \geq \frac{1}{28}
$$

Proof. The proof uses the Paley-Zygmund inequality. Specifically, denote by $Z$ the random variable $R^{T} x$. Then,

$$
\begin{aligned}
\mathbb{E}\left[Z^{2}\right] & =\mathbb{E}\left[\left(\sum_{j=1}^{d} R_{j} x_{j}\right)^{2}\right] \\
& =\mathbb{E}\left[\frac{1}{d}\|x\|_{2}^{2}+\sum_{i=1}^{d} \sum_{j=1}^{d} R_{i} R_{j} x_{i} x_{j} \mathbb{1}\{j \neq i\}\right] \\
& =\frac{1}{d}\|x\|_{2}^{2},
\end{aligned}
$$

where the last step follows from the fact that entries of $R$ are independent with zero-mean. Next, we consider $\mathbb{E}\left[Z^{4}\right]$. Note that the only terms in the expansion of $\left(\sum_{i=1}^{d} R_{i} x_{i}\right)^{4}$ that have nonzero mean are those which have only even powers of entries of $R$. In particular, these are terms of the form $R_{i}^{4} x_{i}^{4}$ 
and $R_{i}^{2} R_{j}^{2} X_{i}^{2} X_{j}^{2}$ with distinct $i, j$. Therefore, we have

$$
\begin{aligned}
\mathbb{E}\left[Z^{4}\right] & =\frac{1}{d^{2}} \sum_{i=1}^{d} x_{i}^{4}+\left(\begin{array}{l}
4 \\
2
\end{array}\right) \frac{1}{d^{2}} \sum_{i=1}^{d} \sum_{j=1}^{d} x_{i}^{2} x_{j}^{2} \mathbb{1}\{i \neq j\} \\
& \leq \frac{1}{d^{2}}\left(\|x\|_{4}^{4}+6\|x\|_{2}^{4}\right) \\
& \leq \frac{7\|x\|_{2}^{4}}{d^{2}}
\end{aligned}
$$

where the final inequality uses $\|x\|_{4} \leq\|x\|_{2}$. Therefore, by the Paley-Zygmund inequality, for $\nu \in(0,1)$,

$$
\mathbb{P}\left(Z^{2}>\nu \mathbb{E}\left[Z^{2}\right]\right) \geq(1-\nu)^{2} \frac{\mathbb{E}\left[Z^{2}\right]^{2}}{\mathbb{E}\left[Z^{4}\right]} \geq \frac{(1-\nu)^{2}}{7}
$$

The claim follows by setting $\nu=1 / 2$.

We are now in a position to complete the proof of Theorem 2. We use the distributed test for $d=1$ from Theorem 1 to build a test for a general $d$. Specifically, we replace the $d$-dimensional observations $X_{1}, \ldots, X_{n}$ of $\mathcal{P}_{1}$ with one-dimensional $\widetilde{X}_{1}, \ldots, \widetilde{X}_{n}$ given by $\widetilde{X}_{t}=R^{T} X_{t}, 1 \leq t \leq n$, where $R$ is a random vector generated as in Lemma 11 . Note that $\left(\widetilde{X}_{t}, Y_{t}\right)_{t=1}^{n}$ are i.i.d. with

$$
\begin{aligned}
\mathbb{E}\left[Y_{1} \widetilde{X}_{1} \mid R\right] & =\mathbb{E}\left[\left(\rho^{T} X_{1}+\sqrt{1-\|\rho\|_{2}^{2}} Z_{1}\right)\left(R^{T} X_{1}\right) \mid R\right] \\
& =\rho^{T} \mathbb{E}\left[X_{1} X_{1}^{T}\right] R \\
& =\rho^{T} R .
\end{aligned}
$$

Thus, by Lemma 11 ,

$$
\mathrm{P}_{R}\left(\left\{r:\left|\mathbb{E}\left[Y_{1} \widetilde{X}_{1} \mid R=r\right]\right| \geq \frac{\|\rho\|_{2}}{\sqrt{2 d}}\right\}\right) \geq \frac{1}{28}
$$

Denoting $\mathcal{G}:=\left\{r:\left|\mathbb{E}\left[Y_{1} \widetilde{X}_{1} \mid R=r\right]\right| \geq \frac{\|\rho\|_{2}}{\sqrt{2 d}}\right\}$ and $\widetilde{\rho}(r):=\left|\mathbb{E}\left[Y_{1} \widetilde{X}_{1} \mid R=r\right]\right|$, for every $r \in \mathcal{G}$ we have

$$
\begin{aligned}
& \widetilde{\rho}(r) \geq \tau / \sqrt{2 d} \text { under } \mathcal{H}_{0}^{d}, \\
& \widetilde{\rho}(r)=0 \text { under } \mathcal{H}_{1}^{d} .
\end{aligned}
$$

Also, in the test we construct for the $d$-dimensional case, we invoke a 1-interactive $(\ell, 1 / 56,1 / 112, \tau / \sqrt{2 d})$ test $T_{1}$ for the one-dimensional correlation testing problem $\widetilde{\rho}(r) \geq \tau / \sqrt{2 d}$ versus $\widetilde{\rho}(r)=0$ with

$$
\ell \leq \frac{c d}{\tau^{2}},
$$


for an appropriate constant $c$, as guaranteed by Theorem 1 .

Next, consider the test for $\mathcal{H}_{0}^{d}$ versus $\mathcal{H}_{1}^{d}$ that samples $R$ from shared randomness executes the aforementioned test $T_{1}$ for $\mathcal{H}_{0}^{d}$ versus $\mathcal{H}_{1}^{d}$ the one-dimensional problem $\widetilde{\rho}(R) \geq \tau / \sqrt{2 d}$ versus $\widetilde{\rho}(R)=0$. We make the observation that $\widetilde{\rho}(R)=0$ almost surely for $R$, when $\rho=0$. Thus, the missed detection probability for the one-dimensional test remains unchanged. However, a false alarm may be raised when $R \notin \mathcal{G}$ or when the one-dimensional test raises a false alarm. It follows that for this test

$$
\mathbb{P}_{\mathcal{H}_{0}^{d}}\left(\text { Declare } \mathcal{H}_{1}^{d}\right) \leq \frac{1}{56}+\mathbb{P}(R \notin \mathcal{G}) \leq \frac{55}{56}
$$

and

$$
\mathbb{P}_{\mathcal{H}_{1}^{d}}\left(\text { Declare } \mathcal{H}_{0}^{d}\right) \leq \frac{1}{112}
$$

whereby it constitutes a $\left(c d / \tau^{2}, 55 / 56,1 / 112, \tau\right)$-test for the $d$-dimensional correlation testing problem.

Thus, we have obtained our desired test with constant probability of error guarantees. Theorem 2 follows by using this test along with Lemma 10.

\section{PROOF OF LOWER BOUNDS FOR $r=1$}

We begin by deriving lower bounds for the one-dimensional problem. Our lower bounds involve the notions of hypercontractivity and reverse hypercontractivity $(c f .[26],[35],[25],[27])$, which we define first.

For $1 \leq q \leq p<\infty$, a pair of random variables $(X, Y)$ is $(p, q)$-hypercontractive if for all $\mathbb{R}$-valued functions $f$ of $X$ and $g$ of $Y$,

$$
\mathbb{E}[|f(X) g(Y)|] \leq\|f(X)\|_{p^{\prime}}\|g(Y)\|_{q}
$$

where $p^{\prime}=p /(p-1)$ is the Hölder conjugate of $p$. Similarly, for $1 \geq q>p$, a pair of random variables $(X, Y)$ is $(p, q)$ - reverse hypercontractive if for all $\mathbb{R}$-valued functions $f$ of $X$ and $g$ of $Y$,

$$
\mathbb{E}[|f(X) g(Y)|] \geq\|f(X)\|_{p^{\prime}}\|g(Y)\|_{q}
$$

The set of all $(p, q)$ for which $(X, Y)$ is $(p, q)$-hypercontractive and $(p, q)$-reverse hypercontractive, respectively, are called the hypercontractivity ribbon and the reverse hypercontractivity ribbon of $(X, Y)$. The following tensorization property of the hypercontractivity and the reverse hypercontractivity ribbon is well known. 
Lemma 12 (Tensorization [35] [27]). For $p \geq 1$, define

$$
q_{p}(X, Y)=\inf \{q:(X, Y) \text { is }(p, q) \text {-hypercontractive }\}
$$

and $r_{p}(X, Y)=q_{p}(X, Y) / p$. If $\left(X_{i}, Y_{i}\right)_{i=1}^{n}$ are independent, then

$$
r_{p}\left(X^{n}, Y^{n}\right)=\max _{1 \leq i \leq n} r_{p}\left(X_{i}, Y_{i}\right)
$$

Furthermore, for $p \leq 1$, define

$$
q_{p}(X, Y)=\sup \{q:(X, Y) \text { is }(p, q) \text {-reverse hypercontractive }\}
$$

and $s_{p}(X, Y)=q_{p}(X, Y) / p$. If $\left(X_{i}, Y_{i}\right)_{i=1}^{n}$ are independent, then

$$
s_{p}\left(X^{n}, Y^{n}\right)=\max _{1 \leq i \leq n} s_{p}\left(X_{i}, Y_{i}\right)
$$

We use the notions of hypercontractivity and reverse hypercontractivity to obtain the change of measure bounds between the joint distribution and the independent distribution, which in turn lead to the following two lower bounds for $C_{1}^{1}(\delta, \varepsilon, \tau)$.

Theorem 13 (Lower bound 1). Given $\delta, \varepsilon \in(0,1)$ and $(p, q)$ such that $1 \leq p^{\prime} \leq q \leq p$ and $(X, Y)$ is $(p, q)$-hypercontractive, the minimum one-way communication for one-dimensional correlation testing $C_{1}^{1}(\delta, \varepsilon, \tau)$ is bounded below as

$$
C_{1}^{1}(\delta, \varepsilon, \tau) \geq \frac{p}{q} \log \frac{1}{\varepsilon}-p \log \frac{1}{1-\delta}
$$

Proof. For $1 \leq q \leq p$, suppose that $(X, Y)$ is $(p, q)$-hypercontractive, which by Lemma 12 implies that $\left(X^{n}, Y^{n}\right)$ is $(p, q)$-hypercontractive. Furthermore, assume that $p^{\prime} \leq q$ which is the same as $q^{\prime} \leq p$. Then, for any subset $\mathcal{A} \subset \mathcal{X}^{n}$ and $\mathcal{B} \subset \mathcal{Y}^{n}$, we have

$$
\mathrm{P}_{X^{n} Y^{n}}(\mathcal{A} \times \mathcal{B}) \leq \mathrm{P}_{X^{n}}(\mathcal{A})^{\frac{1}{p^{\prime}}} \mathrm{P}_{Y^{n}}(\mathcal{B})^{\frac{1}{q}}
$$

We begin by considering a deterministic test where the shared randomness $U$ is constant. Specifically, given a deterministic $(\ell, \delta, \varepsilon, \tau)$-test $T=(f, g)$, denoting $2^{3} L=2^{\ell}$, let $\mathcal{A}_{i}=f^{-1}(i)$ for $i=1, \ldots, L$. Then, $\left\{\mathcal{A}_{1}, \ldots, \mathcal{A}_{L}\right\}$ constitutes a partition of $\mathcal{X}^{n}$. Further, let $\mathcal{B}_{i}$ denote the set $\left\{\mathbf{y} \in \mathcal{Y}^{n}: g(\mathbf{y}, i)=0\right\}$,

\footnotetext{
${ }^{3}$ With a slight abuse of notation, we denote the one-way communication protocol by a mapping $f$.
} 
namely the set of $\mathbf{y}$ where $\mathcal{P}_{2}$ declares $\mathcal{H}_{0}^{1}$ upon receiving $i$ from $\mathcal{P}_{1}$. It follows that

$$
\begin{aligned}
1-\delta & \leq \sum_{i=1}^{L} \mathrm{P}_{X^{n} Y^{n}}\left(\mathcal{A}_{i} \times \mathcal{B}_{i}\right) \\
& \leq \sum_{i=1}^{L} \mathrm{P}_{X^{n}}\left(\mathcal{A}_{i}\right)^{\frac{1}{p^{\prime}}} \mathrm{P}_{Y^{n}}\left(\mathcal{B}_{i}\right)^{\frac{1}{q}}
\end{aligned}
$$

where the previous inequality uses 10 . Upon bounding the right-side using Hölder's inequality, we get

$$
\begin{aligned}
1-\delta & \leq \sum_{i=1}^{L}\left(\mathrm{P}_{X^{n}}\left(\mathcal{A}_{i}\right) \mathrm{P}_{Y^{n}}\left(\mathcal{B}_{i}\right)\right)^{\frac{1}{q}} \mathrm{P}_{X^{n}}\left(\mathcal{A}_{i}\right)^{\frac{1}{p^{\prime}}-\frac{1}{q}} \\
& \leq\left(\sum_{i=1}^{L} \mathrm{P}_{X^{n}}\left(\mathcal{A}_{i}\right) \mathrm{P}_{Y^{n}}\left(\mathcal{B}_{i}\right)\right)^{\frac{1}{q}}\left(\sum_{i=1}^{L} \mathrm{P}_{X^{n}}\left(\mathcal{A}_{i}\right)^{q^{\prime}\left(\frac{1}{p^{\prime}}-\frac{1}{q}\right)}\right)^{\frac{1}{q^{\prime}}} \\
& \leq \varepsilon^{\frac{1}{q}}\left(\sum_{i=1}^{L} \mathrm{P}_{X^{n}}\left(\mathcal{A}_{i}\right)^{q^{\prime}\left(\frac{1}{p^{\prime}}-\frac{1}{q}\right)}\right)^{\frac{1}{q^{\prime}}}
\end{aligned}
$$

where the previous inequality uses the requirement $\mathbb{P}_{\mathcal{H}_{1}^{1}}\left(\right.$ Declare $\left.\mathcal{H}_{0}^{1}\right) \leq \varepsilon$. Noting that $q^{\prime}\left(1 / p^{\prime}-1 / q\right)=$ $1-q^{\prime} / p$, the assumption $q^{\prime} \leq p$ and Hölder's inequality imply

$$
\sum_{i=1}^{L} \mathrm{P}_{X^{n}}\left(\mathcal{A}_{i}\right)^{q^{\prime}\left(\frac{1}{p^{\prime}}-\frac{1}{q}\right)} \leq L^{\frac{q^{\prime}}{p}}
$$

Combining the bounds above, we get

$$
1-\delta \leq \varepsilon^{\frac{1}{q}} L^{\frac{1}{p}}
$$

which completes the proof.

When shared randomness is available, we follow the procedure above for the deterministic test obtained by conditioning on the shared randomness $V$; let $\left(\mathcal{A}_{i}^{V}, \mathcal{B}_{i}^{V}\right), 1 \leq i \leq L$, denote the counterpart of $\left(\mathcal{A}_{i}, \mathcal{B}_{i}\right)$ above for shared randomness $V$. Proceeding as before, we have

$$
\begin{aligned}
1-\delta & \leq \mathbb{E}\left[\left(\sum_{i=1}^{L} \mathrm{P}_{X^{n}}\left(\mathcal{A}_{i}^{V}\right) \mathrm{P}_{Y^{n}}\left(\mathcal{B}_{i}^{V}\right)\right)^{\frac{1}{q}}\left(\sum_{i=1}^{L} \mathrm{P}_{X^{n}}\left(\mathcal{A}_{i}^{V}\right)^{q^{\prime}\left(\frac{1}{p^{\prime}}-\frac{1}{q}\right)}\right)^{\frac{1}{q^{\prime}}}\right] \\
& \leq \mathbb{E}\left[\left(\sum_{i=1}^{L} \mathrm{P}_{X^{n}}\left(\mathcal{A}_{i}^{V}\right) \mathrm{P}_{Y^{n}}\left(\mathcal{B}_{i}^{V}\right)\right)^{\frac{1}{q}}\right] L^{\frac{1}{p}} .
\end{aligned}
$$

It follows from Jensen's inequality that

$$
1-\delta \leq \mathbb{E}\left[\sum_{i=1}^{L} \mathrm{P}_{X^{n}}\left(\mathcal{A}_{i}^{V}\right) \mathrm{P}_{Y^{n}}\left(\mathcal{B}_{i}^{V}\right)\right]^{\frac{1}{q}} L^{\frac{1}{p}}
$$




$$
\leq \varepsilon^{\frac{1}{q}} L^{\frac{1}{p}}
$$

which completes the proof of Theorem 13 .

Theorem 14 (Lower bound 2). Given $\delta, \varepsilon \in(0,1)$ and $(p, q)$ such that $1 \geq q \geq 0 \geq q^{\prime} \geq p$ and $(X, Y)$ is $(p, q)$-reverse hypercontractive, the minimum one-way communication for one-dimensional correlation testing $C_{1}^{1}(\delta, \varepsilon, \tau)$ is bounded below as

$$
C_{1}^{1}(\delta, \varepsilon, \tau) \geq \frac{p}{q} \log \frac{1}{1-\varepsilon}-p \log \frac{1}{\delta}
$$

Proof. For $1 \geq q>p$, suppose that $(X, Y)$ is $(p, q)$-reverse hypercontractive, which with Lemma 12 implies that $\left(X^{n}, Y^{n}\right)$ is $(p, q)$-reverse hypercontractive. Furthermore, assume that $q^{\prime} \geq p$. Then, for any subset $\mathcal{A} \subset \mathcal{X}^{n}$ and $\mathcal{B} \subset \mathcal{Y}^{n}$, for $0 \leq \theta \leq 1$ we have

$$
\mathrm{P}_{X^{n} Y^{n}}(\mathcal{A} \times \mathcal{B})^{\theta} \geq \mathrm{P}_{X^{n}}(\mathcal{A})^{\theta\left(\frac{p-1}{p}\right)} \mathrm{P}_{Y^{n}}(\mathcal{B})^{\theta \frac{1}{q}}
$$

We only provide a proof for deterministic tests; the extension to the case when shared randomness is used can be completed as in the proof of Theorem 13 . Given a deterministic $(\ell, \delta, \varepsilon, \tau)$-test $T=(f, g)$, let $\mathcal{A}_{i}=f^{-1}(i)$ for $i=1, \ldots, L=2^{\ell}$, and let $\mathcal{B}_{i}$ denote the set $\left\{\mathbf{y} \in \mathcal{Y}^{n}: g(\mathbf{y}, i)=0\right\}$. It follows that

$$
\begin{aligned}
1-\varepsilon & \leq \sum_{i=1}^{L} \mathrm{P}_{X^{n}}\left(\mathcal{A}_{i}\right) \mathrm{P}_{Y^{n}}\left(\mathcal{B}_{i}\right) \\
& \leq \sum_{i=1}^{L} \mathrm{P}_{X^{n}}\left(\mathcal{A}_{i}\right)^{1-\theta\left(\frac{p-1}{p}\right)} \mathrm{P}_{Y^{n}}\left(\mathcal{B}_{i}\right)^{1-\frac{\theta}{q}} \mathrm{P}_{X^{n} Y^{n}}\left(\mathcal{A}_{i} \times \mathcal{B}_{i}\right)^{\theta},
\end{aligned}
$$

where the previous inequality uses (12). Upon bounding the right-side using Hölder's inequality, we get

$$
\begin{aligned}
1-\varepsilon & \leq\left(\sum_{i=1}^{L}\left(\mathrm{P}_{X^{n}}\left(\mathcal{A}_{i}\right)^{1-\theta\left(\frac{p-1}{p}\right)} \mathrm{P}_{Y^{n}}\left(\mathcal{B}_{i}\right)^{1-\frac{\theta}{q}}\right)^{\frac{1}{1-\theta}}\right)^{1-\theta}\left(\sum_{i=1}^{L} \mathrm{P}_{X^{n} Y^{n}}\left(\mathcal{A}_{i} \times \mathcal{B}_{i}\right)\right)^{\theta} \\
& \leq\left(\sum_{i=1}^{L} \mathrm{P}_{X^{n}}\left(\mathcal{A}_{i}\right)^{1+\left(\frac{\theta}{p(1-\theta)}\right)} \mathrm{P}_{Y^{n}}\left(\mathcal{B}_{i}\right)^{\frac{q-\theta}{q(1-\theta)}}\right)^{1-\theta} \delta^{\theta}
\end{aligned}
$$

where the previous inequality uses the requirement $\mathbb{P}_{\mathcal{H}_{0}^{1}}\left(\right.$ Declare $\left.\mathcal{H}_{1}^{1}\right) \leq \delta$. Choosing $\theta=q$, the assumption $q^{\prime} \geq p$ together with Hölder's inequality implies

$$
\left(\sum_{i=1}^{L} \mathrm{P}_{X^{n}}\left(\mathcal{A}_{i}\right)^{1+\frac{q}{p(1-q)}}\right)^{1-q} \leq L^{\frac{-q}{p}} .
$$

Combining the bounds above, we get

$$
1-\varepsilon \leq \delta^{q} L^{\frac{-q}{p}},
$$


which completes the proof.

To obtain tight lower bounds for one-dimensional $X$ and $Y$ jointly Gaussian, we need to optimize our lower bounds over the entire hypercontractivity and reverse hypercontractivity ribbon. We rely on the following characterizations of the hypercontractivity and the reverse hypercontractivity ribbons.

Lemma 15 (cf. [23]). Let $X$ and $Y$ be one-dimensional with joint distribution given by (1). For $1 \leq$ $q \leq p,(X, Y)$ is $(p, q)$-hypercontractive if and only if

$$
\frac{q-1}{p-1} \geq \rho^{2}
$$

Furthermore, for $1 \geq q \geq p,(X, Y)$ is $(p, q)$-reverse hypercontractive if and only if

$$
\frac{1-q}{1-p} \geq \rho^{2}
$$

The next corollary is obtained by maximizing the right-sides of (9) and (11), respectively, over the set of $(p, q)$ satisfying (13) and (14); the upper bound is from Theorem 1 .

Corollary 16. For $0<\tau \leq 1$,

1) for $\delta \leq 1$ and $\varepsilon$ such that $\delta+\varepsilon^{\frac{1-\tau}{1+\tau}} \leq 1$,

$$
C_{1}^{1}(\delta, \varepsilon, \tau)=\frac{1}{\tau^{2}} \log \frac{1}{\varepsilon}+\Theta_{\delta}\left(\sqrt{\log \frac{1}{\varepsilon}}\right) ;
$$

2) for $\delta, \varepsilon \in(0,1)$,

$$
C_{1}^{1}(\delta, \varepsilon, \tau)=\frac{1-\tau^{2}}{\tau^{2}} \log \frac{1}{\delta}+\Theta_{\varepsilon}\left(\sqrt{\log \frac{1}{\delta}}\right),
$$

where the notation $\Theta_{x}$ denotes that the constant implied by $\Theta$ depends on $x$.

Proof. Assume first that $\varepsilon^{\frac{1-\tau}{1+\tau}} \leq 1-\delta$. Using the characterization in Lemma 15 . $(X, Y)$ is $(p, q)$ hypercontractive for any $p$ and $q$ satisfying

$$
\begin{aligned}
& p=1+w, \\
& q=1+\tau^{2} w,
\end{aligned}
$$

for any $w \geq 0$. Inserting this choice of $(p, q)$ in the lower bound of Theorem 13 , we get for any $(\ell, \delta, \varepsilon, \tau)$-test that

$$
\ell \geq \frac{1+w}{1+\tau^{2} w} \log \frac{1}{\varepsilon}-(1+w) \log \frac{1}{1-\delta}
$$

For brevity, we denote $\xi:=\log (1-\delta) / \log \varepsilon$; our assumption $\varepsilon^{\frac{1-\tau}{1+\tau}} \leq 1-\delta$ is equivalent to $\xi \leq$ 
$(1-\tau) /(1+\tau)$. To obtain the tightest lower bound, we maximize $(1+w) /\left(1+\tau^{2} w\right)-\xi(1+w)$ over $w \geq 0$. The maximum is obtained at $w^{*}$ given by

$$
w^{*}=\frac{1}{\tau^{2}}\left(\sqrt{\frac{1-\tau^{2}}{\xi}}-1\right),
$$

provided $\xi \leq 1-\tau^{2}$, which holds since $1-\tau^{2} \geq(1-\tau) /(1+\tau)$. Furthermore, the corresponding optimal $p^{*}$ and $q^{*}$ satisfy $p^{* \prime} \leq q^{*}$ if and only if

$$
\tau^{2} \leq\left(\sqrt{\frac{1-\tau^{2}}{\xi}}-1\right)^{2}
$$

which is satisfied when $\xi \leq(1-\tau) /(1+\tau)$. Thus,

$$
\begin{aligned}
\ell & \geq \log \frac{1}{\varepsilon}\left(\frac{1+w^{*}}{1+\tau^{2} w^{*}}-\xi\left(1+w^{*}\right)\right) \\
& =\frac{1}{\tau^{2}}\left(\sqrt{\log \frac{1}{\varepsilon}}-\sqrt{\left(1-\tau^{2}\right) \log \frac{1}{1-\delta}}\right)^{2} .
\end{aligned}
$$

The first part of Corollary 16 follows from 15 and Theorem 1

To get the second part of Corollary 16 , we obtain a replacement for 15 using the reverse hypercontractivity part of Lemma 15 . Specifically, $(X, Y)$ is $(p, q)$-reverse hypercontractive for any $p$ and $q$ satisfying

$$
\begin{aligned}
& p=1-w, \\
& q=1-\tau^{2} w,
\end{aligned}
$$

for any $\frac{1}{\tau^{2}} \geq w \geq 0$ since $q$ must be greater than or equal to 0 . Inserting this choice of $(p, q)$ in the lower bound of Theorem 14, we get for any $(\ell, \delta, \varepsilon, \tau)$-test that

$$
\ell \geq \frac{1-w}{1-\tau^{2} w} \log \frac{1}{1-\varepsilon}-(1-w) \log \frac{1}{\delta}
$$

We maximize the right-side of the above inequality subject to $w \leq \frac{1}{\tau^{2}}$. The maximum is obtained at $w^{*}$ given by

$$
w^{*}=\frac{1}{\tau^{2}}\left(1-\sqrt{\frac{\left(1-\tau^{2}\right) \log \frac{1}{1-\varepsilon}}{\log \frac{1}{\delta}}}\right) .
$$

Note that $w^{*} \leq \frac{1}{\tau^{2}}$ is satisfied for every $\delta$ and $\varepsilon$, and the additional assumption $\varepsilon^{\frac{1-\tau}{1+\tau}} \leq 1-\delta$ of the first 
part of Corollary 16 is not required for the second part. Thus,

$$
\begin{aligned}
\ell & \geq \frac{1-w^{*}}{1-\tau^{2} w^{*}} \log \frac{1}{1-\varepsilon}-\left(1-w^{*}\right) \log \frac{1}{\delta} \\
& =\frac{1}{\tau^{2}}\left(\sqrt{\log \frac{1}{1-\varepsilon}}-\sqrt{\left(1-\tau^{2}\right) \log \frac{1}{\delta}}\right)^{2}
\end{aligned}
$$

which together with Theorem 1 yields the second part of Corollary 16.

Finally, we exploit tensorization property in Lemma 12 to provide a matching lower bound for Theorem 2 in the result below.

Theorem 17. For $0<\tau \leq 1$,

1) for $\delta \in(0,1)$ with $\varepsilon$ such that $\delta+\varepsilon^{\frac{1-\tau}{1+\tau}} \leq 1$, we have

$$
C_{d}^{1}(\delta, \varepsilon, \tau) \geq \frac{d}{\tau^{2}}\left(\sqrt{\log \frac{1}{\varepsilon}}-\sqrt{\left(1-\frac{\tau^{2}}{d}\right) \log \frac{1}{1-\delta}}\right)^{2}
$$

2) for $\delta, \epsilon \in(0,1)$,

$$
C_{d}^{1}(\delta, \varepsilon, \tau) \geq \frac{d}{\tau^{2}}\left(\sqrt{\log \frac{1}{1-\varepsilon}}-\sqrt{\left(1-\frac{\tau^{2}}{d}\right) \log \frac{1}{\delta}}\right)^{2} .
$$

Proof. We consider a different problem where the observation of $\mathcal{P}_{1}$ remains the same but we provide more information to $\mathcal{P}_{2}$. Specifically, $\mathcal{P}_{1}$ observes i.i.d. copies of $X=(X(1), \ldots, X(d))$ and $\mathcal{P}_{2}$ observes i.i.d. copies of $Y=(Y(1), \ldots, Y(d))$ where for $i=1, \ldots, d$,

$$
\mathbb{E}[Y(i) \mid X]=\rho(i) X(i)
$$

Note that in our original problem the observation of $\mathcal{P}_{2}$ are i.i.d. copies of $Y(1)+\ldots+Y(d)$. With this modified observation for $\mathcal{P}_{2}$, we consider the hypothesis testing problem of $\mathcal{H}_{0}^{d}$ versus $\mathcal{H}_{1}^{d}$ as before. Denote by $\widetilde{C}_{d}^{1}(\delta, \varepsilon, \tau)$ the the minimum $\ell$ such that we can find a 1 -interactive $(\ell, \delta, \varepsilon, \tau)$-test for this modified problem. Since the observation for the former problem can be obtained from the latter problem as well, we have

$$
C_{d}^{1}(\delta, \varepsilon, \tau) \geq \widetilde{C}_{d}^{1}(\delta, \varepsilon, \tau)
$$

Furthermore, with $\mathcal{X}=\mathcal{Y}=\mathbb{R}^{d}$, the proof of Theorem 13 applies to the modified problem as well, and we obtain the following bound:

$$
\widetilde{C}_{d}^{1}(\delta, \varepsilon, \tau) \geq \frac{p}{q} \log \frac{1}{\varepsilon}-p \log \frac{1}{1-\delta}
$$

where $\left(X^{n}, Y^{n}\right)$ is $(p, q)$-hypercontractive. By Lemma 12 and Lemma 15 , we can parameterize $p$ and $q$ 


$$
\begin{aligned}
& p=1+w, \\
& q=1+\rho_{\text {max }}^{2} w
\end{aligned}
$$

with $w \geq 0$ and $\rho_{\max }^{2}:=\max _{i=1}^{d} \rho(i)^{2}$. Proceeding as in the proof of Corollary 16 , we get

$$
\widetilde{C}_{d}^{1}(\delta, \varepsilon, \tau) \geq \frac{1}{\rho_{\max }^{2}}\left(\sqrt{\log \frac{1}{\varepsilon}}-\sqrt{\left(1-\rho_{\max }^{2}\right) \log \frac{1}{1-\delta}}\right)^{2} .
$$

Note that we can choose any $\rho$ such that $\|\rho\|_{2} \geq \tau$. Among all such $\rho$ s, the minimum value of $\rho_{\max }$ is attained by $\rho$ with $\rho(i)^{2}=\tau^{2} / d$. Using this value for $\rho$, we get

$$
\widetilde{C}_{d}^{1}(\delta, \varepsilon, \tau) \geq \frac{d}{\tau^{2}}\left(\sqrt{\log \frac{1}{\varepsilon}}-\sqrt{\left(1-\frac{\tau^{2}}{d}\right) \log \frac{1}{1-\delta}}\right)^{2},
$$

which completes the proof of the first part of Theorem 17. The proof of the second part is completed similarly by using the tensorization property of the reverse hypercontractivity ribbon.

\section{LOWER BOUNDS FOR INTERACTIVE COMMUNICATION}

\section{A. Proof of Theorem 5}

Let $T=(\pi, g)$ constitute an $(\ell, \delta, \varepsilon, \tau)$-test. Denote by $P$ the distribution of $\left(Y^{n}, \Pi, V\right)$ under $\mathcal{H}_{0}^{d}$ and by $Q$ the distribution of $\left(Y^{n}, \Pi, V\right)$ under $\mathcal{H}_{1}^{d}$.

Then,

$$
\begin{aligned}
& P\left(g\left(Y^{n}, \Pi, V\right)=1\right) \leq \delta, \\
& Q\left(g\left(Y^{n}, \Pi, V\right)=0\right) \leq \varepsilon .
\end{aligned}
$$

Denoting $P\left(g\left(Y^{n}, \Pi, V\right)=0\right)$ and $Q\left(g\left(Y^{n}, \Pi, V\right)=0\right)$ by $p_{0}$ and $p_{1}$, respectively, let $P_{i}$ denote the Bernoulli distribution with parameter $p_{i}, i=0,1$. Then, by the data processing inequality applied using the channel $\mathbb{1}\left\{g\left(Y^{n}, \Pi, V\right)=0\right\}$, we have

$$
\begin{aligned}
D(P \| Q) & \geq D\left(P_{0} \| P_{1}\right) \\
& \geq p_{0} \log \frac{1}{p_{1}}-1 \\
& \geq(1-\delta) \log \frac{1}{\varepsilon}-1,
\end{aligned}
$$


where we used the bound $h\left(p_{0}\right) \leq 1$ and $h(\cdot)$ denotes the binary entropy function. Furthermore, by Lemma 6 we have $D(P \| Q) \leq \rho_{\max }^{2} \ell$, which with the previous bound gives

$$
\ell \geq \frac{1}{\rho_{\max }^{2}}\left((1-\delta) \log \frac{1}{\varepsilon}-1\right) .
$$

By choosing $\rho$ such that $\|\rho\|_{2} \geq \tau$ and $\rho_{\max }$ is maximized, namely by choosing $\rho(i)^{2}=\tau^{2} / d$ for every $1 \leq i \leq d$, we get the desired bound

$$
\ell \geq \frac{d}{\tau^{2}}\left[(1-\delta) \log \frac{1}{\varepsilon}-1\right]
$$

\section{B. Lower Bound for Estimation}

We provide lower bounds for the estimation error using Fano's method. Using the Gilbert-Varshamov construction (see, for instance, [36, Problem 5.5]) we can find $m \geq 2^{d(1-h(1 / 4))} \geq 2^{d / 6}$ vectors $u_{1}, \ldots, u_{m} \in\{-1,+1\}^{d}$ such that $\bigsqcup^{4} d_{H}\left(u_{i}, u_{j}\right) \geq d / 8$ for every $i \neq j$. Therefore, the vectors $\rho_{i}:=\frac{\Delta}{\sqrt{d}} \cdot u_{i}$, $1 \leq i \leq m$ satisfy

$$
\begin{aligned}
\min _{i, j \in[m]: i \neq j}\left\|\rho_{i}-\rho_{j}\right\|_{2}^{2} & =\frac{4 \Delta^{2} d_{H}\left(u_{i}, v_{i}\right)}{d} \geq \frac{\Delta^{2}}{2}, \\
\max _{i} \rho_{j}(i)^{2} & =\frac{\Delta^{2}}{d}, \quad \forall 1 \leq j \leq m .
\end{aligned}
$$

Consider an $r$-interactive $(\ell, \tau)$-estimate $(\pi, \widehat{\rho})$. We use the estimator $\widehat{\rho}$ to resolve between the hypotheses $\mathcal{H}_{j}, j \in[m]$ where under $\mathcal{H}_{j}, X \in \mathbb{R}^{d}$ and $Y \in \mathbb{R}$ are jointly Gaussian and

$$
\mathbb{E}[Y(i) \mid X]=\rho_{j}(i) X(i), i \in[d]
$$

Consider the test which declare $5 \mathcal{H}_{j}$ if $\left\|\widehat{\rho}-\rho_{j}\right\|_{2}^{2}<\Delta^{2} / 8$; the output is unique since $\left\|\rho_{i}-\rho_{j}\right\|_{2}^{2} \geq \Delta^{2} / 2$ for every $i \neq j$. The probability of error for this test under $\mathbb{P}_{\rho_{j}}$ is bounded above by

$$
\mathbb{P}_{\rho_{j}}\left(\left\|\widehat{\rho}-\rho_{j}\right\|_{2}^{2} \geq \Delta^{2} / 8\right) \leq \frac{8}{\Delta^{2}} \mathbb{E}_{\rho_{j}}\left[\left\|\widehat{\rho}-\rho_{j}\right\|_{2}^{2}\right]
$$

where the inequality is by Markov's inequality. Therefore, denoting by $P_{e}^{*}$ the minimum average probability of error for this hypothesis testing problem under uniform prior on the hypotheses, we get from (2) that

$$
\tau^{2} \geq \max _{j \in[m]} \frac{\Delta^{2}}{8} \mathbb{P}_{\rho_{j}}\left(\left\|\widehat{\rho}-\rho_{j}\right\|_{2}^{2} \geq \Delta^{2} / 8\right)
$$

${ }^{4}$ We denote by $d_{H}(u, v)$ the Hamming distance between $u$ and $v$.

${ }^{5}$ In the remainder of this proof, with an abuse of notation, we denote the random variable $\widehat{\rho}\left(Y^{n}, \Pi, V\right)$ by $\widehat{\rho}$. 


$$
\begin{aligned}
& \geq \frac{\Delta^{2}}{8 m} \sum_{i=1}^{m} \mathbb{P}_{\rho_{j}}\left(\left\|\widehat{\rho}-\rho_{j}\right\|_{2}^{2} \geq \Delta^{2} / 8\right) \\
& \geq \frac{\Delta^{2}}{8} P_{e}^{*}
\end{aligned}
$$

By Fano's inequality, we have

$$
P_{e}^{*} \geq 1-\frac{C(W)+1}{\log m}
$$

where $W$ denotes the channel with input $j \in\{1, \ldots, m\}$ and output $\left(Y^{n}, \Pi, V\right)$ with distribution corresponding to the correlation $\rho_{j}$ between $X^{n}$ and $Y^{n}$, and $C(W)$ denotes the capacity of channel $W$. Recall the well-known bound

$$
C(W) \leq \min _{Q} \max _{j} D(W(\cdot \mid j) \| Q)
$$

We use this bound with $Q$ chosen to be the distribution of $\left(Y^{n}, \Pi, V\right)$ when the correlation between $X$ and $Y$ is $\rho=0$.

Then, by Lemma 6 we have

$$
D(W(\cdot \mid j) \| Q) \leq \max _{1 \leq i \leq d} \rho_{j}(i)^{2} \ell=\frac{\Delta^{2} \ell}{d} .
$$

Combining the bounds above yields

$$
\tau^{2} \geq \frac{\Delta^{2}}{8}\left(1-\frac{6\left(\Delta^{2} \ell / d+1\right)}{d}\right)
$$

In particular, for $d \geq 12$, setting $\Delta^{2} \ell / d^{2}=1 / 24$ gives $\ell \geq \frac{d^{2}}{768 \tau^{2}}$. Note that for $d<12$, for an appropriate constant $c$, the lower bound $\ell \geq c d^{2} / \tau^{2}$ holds since we already have an $\Omega\left(d / \tau^{2}\right)$ lower bound for the testing problem. This completes the proof.

\section{EXTENSIONS AND DISCUSSION}

We conclude with a discussion on various extensions of our result, and state some of these extensions without proof (the proofs are very similar to the others in the paper).

First, we note that while the hypercontractivity based lower bound yields a tight dependence on $\delta$ or $\varepsilon$ separately in Corollary 16, it does not characterize the joint dependence on $\delta$ and $\varepsilon$ simultaneously. Interestingly, when we allow $\delta>1 / 2$ and have $\varepsilon$ small, such a joint characterization is possible. Specifically, for $d=1$, consider the simple binary hypothesis problem of correlation $\rho$ versus correlation 0 . The test we use in Theorem 8 for resolving between $\mathcal{H}_{0}^{+}$and $\mathcal{H}_{1}^{1}$ with a different choice for $\theta$ and $r$ yields a joint characterization of one-way minimum communication needed for this problem (see [1]). Interestingly, the overall communication is below $\left(1 / \rho^{2}\right) \max \{\log 1 / \varepsilon, \log 1 / \delta\}$. 
Theorem 18. For $d=1,0<\rho \leq 1, \delta \in(1 / 2,1)$, and $\varepsilon$ such that $\delta+\varepsilon^{\frac{1-\tau}{1+\tau}} \leq 1$, the minimum one-way communication needed to test if correlation is $\rho$ or 0 is given by

$$
\frac{1}{\rho^{2}} \cdot\left(\sqrt{\log \frac{1}{\varepsilon}}-\sqrt{\left(1-\rho^{2}\right) \log \frac{1}{1-\delta}}\right)^{2}+O\left(\sqrt{\log \frac{1}{\varepsilon} \log \frac{1}{1-\delta}}\right) .
$$

In another direction, we can consider the simple binary hypothesis testing problem of $\rho=\rho_{0}$ versus $\rho=\rho_{1}$, where $1>\rho_{0}>\rho_{1}>0$. Once again, by modifying the parameters for the test used in Theorem 8 , we get a generalization of our results for $d=1$ to the case $\rho_{1}>0$. Specifically, in this case, the probability of error requirements as in (4) and (5) yield

$$
\begin{gathered}
1-e^{2^{k} Q(r)}-Q\left(\frac{r\left(\rho_{0}-\theta\right)}{\sqrt{1-\rho_{0}^{2}}}\right)-\eta \geq 1-\delta, \\
2^{k+1} Q(r) Q\left(\frac{r\left(\theta-\rho_{1}\right)}{\sqrt{1-\rho_{1}^{2}}}\right) \leq \epsilon .
\end{gathered}
$$

Proceeding in a similar manner as our earlier analysis and upon setting $\theta \in\left(\rho_{1}, \rho_{0}\right)$ and

$$
r^{2}=\frac{2 \ln 2}{\left(\rho_{0}-\rho_{1}\right)^{2}}\left(\sqrt{\left(1-\rho_{1}^{2}\right) \log \frac{1}{\varepsilon}+\log \ln \frac{3}{\delta}+1}+\sqrt{\left(1-\rho_{0}^{2}\right) \log \frac{3}{\delta}}\right)^{2},
$$

we obtain the following result.

Theorem 19. For $d=1, \delta, \varepsilon \in(0,1), 0<\rho_{1}<\rho_{0}<1$, we can find a distributed test for $\rho=\rho_{0}$ versus $\rho=\rho_{1}$ that uses one-way communication of less than

$$
\frac{1}{\left(\rho_{0}-\rho_{1}\right)^{2}}\left(\sqrt{\left(1-\rho_{1}^{2}\right) \log \frac{1}{\varepsilon}}+\sqrt{\left(1-\rho_{0}^{2}\right) \log \frac{1}{\delta}}\right)^{2}+O\left(\sqrt{\log \frac{1}{\varepsilon}+\log \ln \frac{1}{\delta}} \sqrt{\log \frac{1}{\delta}}\right) \text { bits. }
$$

We note that [13] derived an upper bound for the error-exponent for this problem when communication length per sample is fixed. While the result there was stated for error exponent, the main bound [13, Equation (48)] shows that the one-way communication needed for testing $\rho=\rho_{0}$ versus $\rho=\rho_{1}$ must exceed

$$
\left(\frac{\left(1-\rho_{1}\right)^{2}}{\left(\rho_{0}-\rho_{1}\right)^{2}}-1\right)\left(\max \left\{(1-\delta) \log \frac{1}{\varepsilon},(1-\varepsilon) \log \frac{1}{\delta}\right\}-1\right)
$$

which almost matches the communication requirement for our scheme. However, we do not account for the number of samples in our scheme, and it may not attain the upper bound on the error exponent in [13].

We close with the remark that extending our results to the case when the basis used to express $\mathbb{E}\left[Y_{1} \mid X_{1}=x_{1}\right]$ as a linear function of $x_{1}$ is unknown is practically relevant and an interesting open 
problem. It will account for testing for correlation in some unknown "feature representation", and not along fixed features as in our current setting.

\section{ACKNOWLEDGEMENTS}

The authors thank Ofer Shayevitz and Uri Hadar for useful discussions and Prathamesh Mayekar for suggesting the use of median trick in the proof of upper bound for general $d$.

\section{REFERENCES}

[1] K. R. Sahasranand and H. Tyagi, "Extra samples can reduce the communication for independence testing," in 2018 IEEE International Symposium on Information Theory (ISIT). IEEE, 2018, pp. 2316-2320.

[2] R. Ahlswede and I. Csiszár, "Hypothesis testing with communication constraints," IEEE Trans. Inf. Theory, vol. 32, no. 4, pp. 533-542, July 1986.

[3] T.S.Han, "Hypothesis testing with multiterminal data compression," vol. 33, no. 6, pp. 759-772, November 1987.

[4] T. S. Han and S. Amari, "Statistical inference under multiterminal data compression," IEEE Trans. Inf. Theory, vol. 44, no. 6, pp. 2300-2324, October 1998.

[5] H. M. H. Shalaby and A. Papamarcou, "Multiterminal detection with zero-rate data compression," IEEE Trans. Inf. Theory, vol. 38, no. 2, pp. 254-267, March 1992.

[6] Y. Xiang and Y. H. Kim, "Interactive hypothesis testing with communication constraints," in 50th Annual Allerton Conference on Communication, Control, and Computing, October 2012, pp. 1065-1072.

[7] _ - "Interactive hypothesis testing against independence," in 2013 IEEE International Symposium on Information Theory, July 2013, pp. 2840-2844.

[8] W. Zhao and L. Lai, "Distributed testing against independence with multiple terminals," in 52nd Annual Allerton Conference on Communication, Control, and Computing, September 2014, pp. 1246-1251.

[9] M. Wigger and R. Timo, "Testing against independence with multiple decision centers," in International Conference on Signal Processing and Communications (SPCOM), June 2016, pp. 1-5.

[10] G. Katz, P. Piantanida, and M. Debbah, "Collaborative distributed hypothesis testing with general hypotheses," in 2016 IEEE International Symposium on Information Theory (ISIT). IEEE, 2016, pp. 1705-1709.

[11] S. Sreekumar and D. Gündüz, "Distributed hypothesis testing over discrete memoryless channels," IEEE Transactions on Information Theory, 2019.

[12] M. S. Rahman and A. B. Wagner, "On the optimality of binning for distributed hypothesis testing," IEEE Transactions on Information Theory, vol. 58, no. 10, pp. 6282-6303, 2012.

[13] U. Hadar, J. Liu, Y. Polyanskiy, and O. Shayevitz, "Error exponents in distributed hypothesis testing of correlations," in 2019 IEEE International Symposium on Information Theory (ISIT). IEEE, 2019, pp. 2674-2678.

[14] _ - "Communication complexity of estimating correlations," in Proceedings of the 51st Annual ACM SIGACT Symposium on Theory of Computing, 2019, pp. 792-803.

[15] A. Andoni, T. Malkin, and N. S. Nosatzki, "Two party distribution testing: Communication and security," in 46th International Colloquium on Automata, Languages, and Programming (ICALP 2019). Schloss Dagstuhl-Leibniz-Zentrum fuer Informatik, 2019.

[16] Y. Dagan and O. Shamir, "Detecting correlations with little memory and communication," in Conference On Learning Theory, 2018, pp. 1145-1198. 
[17] J. Acharya, C. L. Canonne, and H. Tyagi, "Inference under information constraints: Lower bounds from chi-square contraction,” Proceedings of Machine Learning Research vol, vol. 99, pp. 1-15, 2019.

[18] — - "Inference under information constraints ii: Communication constraints and shared randomness," arXiv preprint arXiv:1905.08302, 2019.

[19] J. Acharya, C. Canonne, C. Freitag, and H. Tyagi, "Test without trust: Optimal locally private distribution testing," in Proceedings of Machine Learning Research, ser. Proceedings of Machine Learning Research, K. Chaudhuri and M. Sugiyama, Eds., vol. 89. PMLR, 16-18 Apr 2019, pp. 2067-2076.

[20] J. Acharya, C. L. Canonne, Y. Han, Z. Sun, and H. Tyagi, "Domain compression and its application to randomness-optimal distributed goodness-of-fit," arXiv preprint arXiv:1907.08743, 2019.

[21] U. Hadar and O. Shayevitz, "Distributed estimation of gaussian correlations," IEEE Transactions on Information Theory, vol. 65, no. 9, pp. 5323-5338, 2019.

[22] A. Bonami, "Etudes des coefficients Fourier des fonctiones de $L^{p}(G)$," Ann. Inst. Fourier, vol. 20, no. 2, pp. 335-402, 1970.

[23] L. Gross, "Logarithmic sobolev inequalities,” American Journal of Mathematics, vol. 97, no. 4, pp. 1061-1083, 1975.

[24] W. Beckner, "Inequalities in Fourier analysis," Ann. of Math., vol. 102, no. 1, pp. 159-182, July 1975.

[25] C. Borell, "Positivity improving operators and hypercontractivity," Mathematische Zeitschrift, no. 180, pp. 225-234, 1982.

[26] R. Ahlswede and P. Gacs, "Spreading of sets in product spaces and hypercontraction of the markov operator," Ann. Probab., vol. 4, no. 6, pp. 925-939, December 1976.

[27] E. Mossel, K. Oleszkiewicz, and A. Sen, "On reverse hypercontractivity," Geometric and Functional Analysis, vol. 23, no. 3, pp. 1062-1097, June 2013.

[28] V. Guruswami and J. Radhakrishnan, "Tight bounds for communication-assisted agreement distillation," in Proceedings of the 31st Conference on Computational Complexity, 2016, pp. 6:1-6:17.

[29] R. Ahlswede and I. Csiszár, "Common randomness in information theory and cryptography-part II: CR capacity," IEEE Trans. Inf. Theory, vol. 44, no. 1, pp. 225-240, January 1998.

[30] H. Tyagi and S. Watanabe, "A bound for multiparty secret key agreement and implications for a problem of secure computing," in EUROCRYPT, 2014, pp. 369-386.

[31] — - "Converses for secret key agreement and secure computing," IEEE Trans. Inf. Theory, vol. 61, no. 9, pp. 4809-4827, September 2015.

[32] J. Acharya, C. Daskalakis, and G. Kamath, "Optimal testing for properties of distributions," in Advances in Neural Information Processing Systems 28. Curran Associates, Inc., 2015, pp. 3591-3599.

[33] J. Liu, P. Cuff, and S. Verdú, "Secret key generation with limited interaction," IEEE Trans. Inf. Theory, vol. 63, no. 11, pp. 7358-7381, November 2017.

[34] R. Durrett, Probability: theory and examples. Cambridge university press, 2019, vol. 49.

[35] C. Nair, "Equivalent formulations of hypercontractivity using information measures," Proceedings of International Zürich Seminar on Communications, 2014.

[36] I. Csiszár and J. Körner, Information theory: Coding theorems for discrete memoryless channels. 2nd edition. Cambridge University Press, 2011. 